\title{
MMFO: modified moth flame optimization algorithm for region based RGB color image segmentation
}

\author{
Varshali Jaiswal $^{1}$, Varsha Sharma ${ }^{2}$, Sunita Varma ${ }^{3}$ \\ ${ }_{1,2}^{1}$ School of Information Technology, RGPV, Bhopal, India \\ ${ }^{3}$ Department of Information Technology, SGSITS, RGPV, Indore, India
}

\begin{abstract}
Article Info
Article history:

Received Mar 10, 2019

Revised Aug 16, 2019

Accepted Aug 29, 2019

\section{Keywords:}

Clustering

Genetic approach

Image segmentation

Moth-flame optimization

Particle swarm optimization

ABSTRACT

Region-based color image segmentation is elementary steps in image processing and computer vision. The region-based color image segmentation has faced the problem of multidimensionality. The color image is considered in five-dimensional problems, in which three dimensions in color (RGB) and two dimensions in geometry (luminosity layer and chromaticity layer). In this paper, L*a*b color space conversion has been used to reduce the one dimension and geometrically it converts in the array hence the further one dimension has been reduced. This paper introduced, an improved algorithm modified moth flame optimization (MMFO) algorithm for RGB color image segmentation which is based on bio-inspired techniques. The simulation results of MMFO for region based color image segmentation are performed better as compared to PSO and GA, in terms of computation times for all the images. The experiment results of this method gives clear segments based on the different color and the different number of clusters is used during the segmentation process.
\end{abstract}

Copyright $@ 2020$ Institute of Advanced Engineering and Science. All rights reserved.

\section{Corresponding Author:}

Varshali Jaiswal,

School of Information Technology, University Teaching Department,

Rajiv Gandhi Proudyogiki Vishwavidyalaya (State Technological University of Madhya Pradesh),

Airport Road, Gandhi Nagar, Bhopal-462033, India.

Email: varshalijaiswal@gmail.com

\section{INTRODUCTION}

The digital image segmentation [1] becomes an important field of research to enhance the performance of the identifying object classes. Image segmentation is the digital image processing techniques by which an original image is divided into the different region. Many researchers have used different techniques for image segmentation like region growing, edge detection, threshold-based methods, clustering, etc. Clustering [2] is a method that can group objects or patterns into a predefined number of clusters so that object or patterns share their properties. The cluster can be hierarchical and partitioned. Hierarchical cluster produce a nested series of partitions while partition cluster produces only one partition. In computer vision, image processing problem [3] requires color image segmentation in order to identify a target and cluster the image into segments according to color, motion, texture, etc. Real life applications of color image segmentation in medical image processing are tumors detection from the brain image, surgery by computer system and study of anatomical, etc.

There are many researchers working towards region-based color image segmentation problems. The region-based image segmentation [4] is processed in which image are partition based on properties of their region like color, text, luminosities, etc. Color image segmentation [1] is a key problem in image processing and their applications are endless. There are several methods used to find the color image segmentation such as mean shift algorithm [5] and particle swarm optimization etc. Velayudham et al [6] implemented a method for detecting and classifying brain tumor in medical images. In their research they firstly remove unwanted noise using dual-tree complex wavelet packets and empirical mode decomposition. 
Medical image is segmented using a K-means clustering technique and Cuckoo-neuro fuzzy algorithm used for detection of brain tumor. Puranik [7] utilize a fuzzy system for color classification and image segmentation. The highest fitness value is chosen as the best set of fuzzy rules for image segmentation. Sankari [8] proposed a method which used Glowworm swarm optimization (GSO) and the expectationmaximization (EM) based clustering methods for image segmentation. The proposed approach is compared with the standard Gaussian mixture models (GMM)-EM by using Berkley's image data set. Rand index measure and global consistency error (GCE) are used for measuring the performance algorithm. Ma et al [9] developed novel approach region based color image segmentation for skin lesions detection in medical image. Their approach is a combination of speed function, saturation and color information. The main objective of using that algorithm is to cluster pixel into small segments and pixel in each segment possesses similar characteristics. Yanhui [10] proposed a neutrosophic adaptive mean shift clustering method for color image segmentation. Wang [11] proposed color image segmentation by the pixel classification method using QEMs and TSVM classifier. Cheng [12] proposed a region-growing approach for color image segmentation which is based on 3-D clustering and labeling. The features of the algorithm are that it takes color similarity and spatial proximity as input. The [13] proposed the genetic algorithm and classical c-means clustering algorithm (CMA) based color image segmentation. Li [14] proposed color image segmentation method based on an evolutionary approach to the control system. This method determines the threshold values of HSV range. The disadvantages of this method are that takes times for time-consuming process of segmenting the boundary of the color images. However Belahbib [15] proposed a genetic algorithm based clustering method for color image segmentation. The output of the algorithm is a flexible string length with fitness value. There are many approaches used for the color image segmentation like Rajinikanth [16] which used for multi-level image segmentation. The performance measuring parameter is SSIM, PSNR and CPU time. Amelio [17] investigates a graph-based approach for image segmentation. The results show that the method is applicable for partitioning natural and human scenes. The authors conclude the genetic algorithm can be a very efficient method compare to others.

The rest of the paper is organized as follows: research method is reviewed and describes the detailed information about our proposed algorithm formed by the evolutionary approach in Section 2 . Section 3 gives the simulation and performance of the proposed algorithm and comparatively analysis of the proposed algorithm with previous approaches in the literature. Conclusions and possible future research directions are explored in section 4. The proposed method is tested on three different color RGB test images.

\section{RESEARCH METHOD}

In this section, the color image segmentation problem [18] is illustrated which is defined mathematically by considering image $I(i, j)$ to be segmented into $K$ number of cluster. Where ( $i, j$ ) is represented pixel location. A grouping of the pixel having same color components is termed as color clustering [19]. The color component of each pixel in image is represented by a vector. In RGB image the vector is [R1, B1, G1] where R1, G1, B1 simultaneously represents red, green and blue vector components. The pixel value represented by equation 1 which is given below as

$$
0<=\mathrm{R}<=255,0<=\mathrm{G}<=255,0<=\mathrm{B}<=255
$$

In this paper, image is converted into $\mathrm{L}^{*} \mathrm{a} * \mathrm{~b}$ color model [15], where $\mathrm{a}^{*} \mathrm{~b}^{*}$ component represent the color of pixel. This method convert 3D image into 2D image, which is shown in (2).

$$
[\mathrm{RGB}] \rightarrow[\mathrm{A}, \mathrm{b}]
$$

Assuming that total $\mathrm{N}$ is number of pixel used to represent an image. Then $\mathrm{a}_{1}, \mathrm{a}_{2} \ldots \ldots, \mathrm{a}_{\mathrm{N}}$ and $b_{1}, b_{2}, \ldots b_{N}$ vector are used to represent their color component. The proposed method utilize clustering algorithm for perform grouping of pixel in vector A and B which is shown in (3)

$$
A \vec{A}=\left[a_{1}, a_{2}, \ldots \ldots \ldots a_{n}\right], \vec{B}=\left[b_{1}, b 2, \ldots \ldots, b_{n}\right]
$$

The main objective of generating number of cluster in vector A and B is to find minimum distance between them. The $\mathrm{K}$ is number of cluster that is farmed during the clustering process and each cluster is represented by $a_{c 1}, a_{c 2}, \ldots \ldots a_{c k}$ and $b_{c 1}, b_{c 2}, \ldots \ldots b_{c k}$. The optimization function for the image segmentation is defined by (4)

$$
\operatorname{Min} \sum_{j=1}^{k} \sum_{i=1}^{k} p_{(i, j)} d_{(i, j)}
$$


where $p_{(i . j)}=\{0,1\}$, if $\mathrm{i}^{\text {th }}$ pixel is in the $\mathrm{j}^{\text {th }}$ cluster the $p_{(i . j)}=1$ otherwise $p_{(i . j)}=0$ and $d_{(i, j)}$ is represented by (5).

$$
d_{(i, j)}=\sqrt{\left(a_{i}-a_{j}\right)^{2}+\left(b_{i}-b_{j}\right)^{2}}
$$

\subsection{The proposed algorithm for region-based RGB color image segmentation}

In this section, proposed algorithm is explained for the region based RGB color image segmentation [14]. Region based RGB color images segmentation in which similar data points are grouped together into clusters which is describe Figure 1. El Aziz [20] andn Mirjalili [21] recently proposed a swarm optimization technique, which is inspired by behavior of moth and known as moth flame optimization (MFO) algorithm. In this paper MFO algorithm applied for the region based RGB color image segmentation. This transverse orientation seems useful only when the light is very far. When the light is too close, it directs the spiral path towards the light which is shown in Figure 2. In the proposed MFO algorithm, assumptions are taken to define as candidate solutions are moths and the problem's variables are the position of moths in the space. Therefore, the moths can fly in 1-D, 2-D, 3-D, or hyper dimensional space with respect to their position vectors. By using the position of moths and flames MFO algorithm can be modeled. This is represents the cluster center for $\mathrm{a}^{*} \mathrm{~b}$ space of images. The fitness value given in equation 10 is optimized within boundaries. Lower bouny [Lc1, Lc2,... Lck], and Upper boun [Uc1, Uc2,......Uck] where Upper boundary $(\mathrm{Ui})<=255$, Lower boundary $(\mathrm{Li})=0$, and $\mathrm{i}=1$ to $\mathrm{k}$. The dimensions of moth and flame are different in space. Matrix is used to represent the position of all moth with corresponding fitness value. Similarly the position of the all flame with corresponding fitness value which is describe in Figure 3.

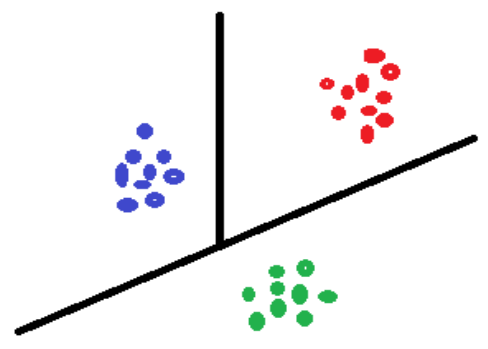

Figure 1. Similar data points grouped together into clusters

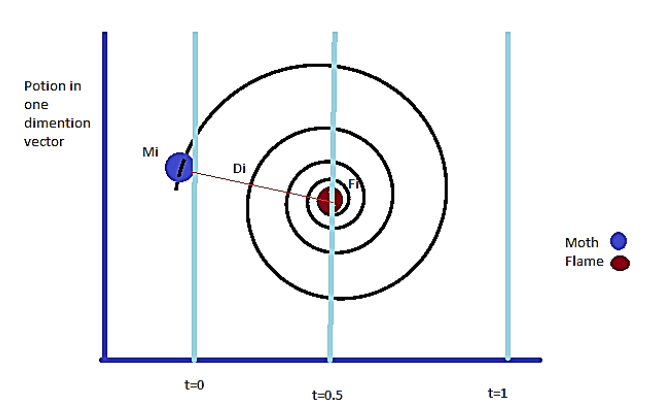

Figure 2. Logarithm spiral space around the Flame and the Moth position with respect to $t$

The population-based MFO algorithm, in which matrix a, is used to describe for position of all Moth and matrix $b$ give fitness value of the corresponding Moth. Where $\mathrm{n}$ are the number of Moths and $\mathrm{d}$ number of dimension variables.

$$
\mathrm{M}=\left[\begin{array}{ccc}
\mathrm{m}_{1,1} & \cdots & \mathrm{m}_{1, \mathrm{~d}} \\
\vdots & \vdots & \vdots \\
\mathrm{m}_{\mathrm{n}, 1} & \cdots & \mathrm{m}_{\mathrm{n}, \mathrm{d}}
\end{array}\right]
$$

(a)
$\mathrm{OM}=\left[\begin{array}{c}\mathrm{OM}_{1} \\ \mathrm{OM}_{2} \\ \vdots \\ \mathrm{OM}_{\mathrm{n}}\end{array}\right]$

(b)
$F=\left[\begin{array}{ccc}F_{1,1} & \cdots & F_{1, d} \\ \vdots & \vdots & \vdots \\ F_{n, 1} & \cdots & F_{n, d}\end{array}\right]$

(c)
$\mathrm{OF}=\left[\begin{array}{c}\mathrm{OF}_{1} \\ \mathrm{OF}_{2} \\ \vdots \\ \mathrm{OF}_{\mathrm{n}}\end{array}\right]$

(d)

Figure 3. Matrixes represent Moth and Flame with corresponding fitness value

The result of fitness function for each Moth is another key component in the proposed algorithm. The matrix c used to represent potation of all Flames and matrix d give fitness value of the corresponding Flame. Matrixes in Figure 3 are used to simulate spiral flying path of Moths. Different parameters used by MFO algorithms are iterations number, number of the predefined clusters and search agents number. The proposed algorithm compared with GA and PSO algorithm. The GA [17, 22] in which first read RGB color image, then for every cluster calculate $\mathrm{L} * \mathrm{a} * \mathrm{~b} *$ factor for each color. 
The Genetic-based clustering algorithm cluster the different color by draw graph of the 'a*'and 'b*' values of pixels that were segmented into different colors. PSO [23] algorithm work based on the behavior of birds to solve the optimization problems. In this algorithm, the particles can more quickly converge to the optimal solution because the global best particle to provide information to other particles and all the update process is to follow the current optimal solution [24, 25]. PSO works as fallows first initialize each particle then for each particle calculate the fitness value and personal best (pBest).

\subsection{Simulation and performance evaluation}

In this section, we simulate the proposed algorithm. The aim of this experiment is to improve the fitness value, segment colour image and plot the graph between $a^{*} b^{*}$ colour labels and decreasing computation time.

\subsubsection{Simulation parameters}

In the process of color segmentation, firstly read RGB image then converted into $\mathrm{L}^{*} \mathrm{a} * \mathrm{~b}$ space. This process reduces one of the dimensions which represent the color information. The $a^{*} b$ space is representing the color component of image and $\mathrm{a}^{*} \mathrm{~b}^{*}$ component are converted into on array. These reduce the two dimensional geometric information into one dimension. This $\mathrm{a}^{*} \mathrm{~b}^{*}$ space information is then clustered using proposed algorithm and each cluster is processed to find the color segment of image. A colour image segmentation method is simulated using Genetic Approach, Particle Swarm Optimization and Moth-flame optimization algorithm for clustering the images. The results are calculated by Matlab R2013a on an Intel(R) Core(TM) i5-6500 CPU @ 3.20 GHz, 4.00 RAM running window 10.

The proposed method is tested on colour RGB test images from https://homepages.cae.wisc.edu/ ece533/images/ (512*512 pixels). Using MATLAB tools understand the colour image segmentation by proposed algorithm and tested on different test images from the database. The typical results for the different image of the colours are shown in Figures 4 and Table 1 represents simulation parameter used by the proposed algorithm. The fitness value of GA, PSO and MFO based clustering algorithm are used to minimize the sum of distances between cluster centre and its members for each cluster.

\subsubsection{Performance metrics}

In this section calculated performance of proposed algorithm and compared with other evolutionary approach. All algorithm used in this paper have the same stopping conditions. In proposed algorithm fitness value and computation time are used to evaluate the performance of RGB image segmentation. The fitness value is showing the color difference in an image which is based on $\mathrm{L}^{*} \mathrm{a}^{*} \mathrm{~b}^{*}$ color luminous. The proposed algorithm is using the concepts of Euclidean distance and $\mathrm{L}^{*} \mathrm{a} * \mathrm{~b}^{*}$ color luminous [1]. The color difference between two colors, $(\mathrm{L} 1 *, \mathrm{a} 1 *, \mathrm{~b} 1 *$ and $\mathrm{L} 2 * \mathrm{a} 2 * \mathrm{~b} 2 *)$ is calculate which is describe in (6) and the performance of proposed algorithm are tabulated in Table 2.

Table 1. Simulation parameter for different Images

\begin{tabular}{|c|c|c|c|}
\hline S.No. & & Parameter & Description \\
\hline 1 & $\begin{array}{l}\text { Parameter } \\
\text { for }\end{array}$ & Image Size & $\begin{array}{c}512 * 512 \\
\text { Pixel }\end{array}$ \\
\hline 2 & algorithm & Image type & PNG Image \\
\hline 3 & & K-Factor & $2,3,4,5$ \\
\hline 4 & & $\begin{array}{l}\text { Maximum no. of } \\
\text { Iteration }\end{array}$ & 200 \\
\hline 5 & GA & $\begin{array}{l}\text { Crossover } \\
\text { Percentage }\end{array}$ & 08 \\
\hline 6 & & Mutation Rate & 0.02 \\
\hline 7 & & Mutation & \\
\hline 8 & PSO & $\begin{array}{c}\text { Percentage } \\
\text { Population Size } \\
\text { (Swarm Size) }\end{array}$ & 0.3 \\
\hline 9 & & Inertia Weight & \\
\hline 10 & & $\begin{array}{l}\text { Damping Ratio } \\
\text { Constriction }\end{array}$ & 0.99 \\
\hline 11 & MFO & $\begin{array}{l}\text { Population Size } \\
\text { (Moth Size) }\end{array}$ & 50 \\
\hline 12 & & $\begin{array}{l}\text { Population Size } \\
\text { (Moth Size) }\end{array}$ & 50 \\
\hline
\end{tabular}

Table 2. The best fitness value obtained from

\begin{tabular}{|c|c|c|c|c|c|}
\hline Image & $\mathrm{k}$ & iteration & $\begin{array}{c}\text { MFO } \\
\text { Fitness value }\end{array}$ & $\begin{array}{c}\text { PSO } \\
\text { Fitness value }\end{array}$ & $\begin{array}{c}\text { GA } \\
\text { Fitness value }\end{array}$ \\
\hline \multirow{20}{*}{ Image 1 } & \multirow{5}{*}{5} & 1 & 223800.6438 & 173704.5129 & 144855.9356 \\
\hline & & 50 & 113745.1509 & 104368.1311 & 104296.7006 \\
\hline & & 100 & 107486.7579 & 104270.4416 & 104271.8102 \\
\hline & & 150 & 105991.2988 & 104270.2473 & 104274.4183 \\
\hline & & 200 & 104270.6937 & 104270.2471 & 104272.1806 \\
\hline & \multirow{5}{*}{4} & 1 & 204536.5125 & 184593.3676 & 218473.9842 \\
\hline & & 50 & 123813.8133 & 119923.7911 & 119921.1493 \\
\hline & & 100 & 119955.7861 & 119919.2042 & 119919.2217 \\
\hline & & 150 & 119919.6327 & 119919.1969 & 119919.2118 \\
\hline & & 200 & 119919.0330 & 119919.1969 & 119919.2112 \\
\hline & \multirow{5}{*}{3} & 1 & 240628.9909 & 208617.5766 & 189116.8434 \\
\hline & & 50 & 154692.4700 & 154173.2727 & 154173.0245 \\
\hline & & 100 & 154176.6397 & 154172.0967 & 154172.1260 \\
\hline & & 150 & 154172.1028 & 154172.0962 & 154172.1231 \\
\hline & & 200 & 154172.0962 & 154172.0962 & 154172.1215 \\
\hline & \multirow{5}{*}{2} & 1 & 271475.3318 & 246706.8284 & 263679.3727 \\
\hline & & 50 & 236151.1116 & 234626.4496 & 234626.2914 \\
\hline & & 100 & 234626.2796 & 234626.2687 & 234626.2687 \\
\hline & & 150 & 234626.2687 & 234626.2687 & 234626.2687 \\
\hline & & 200 & 234626.2687 & 234626.2687 & 234626.2687 \\
\hline
\end{tabular}




$$
d(x, y)=d(y, x)=\sqrt{\left(y_{1}-x_{1}\right)^{2}+\left(y_{2}-x_{2}\right)^{2}+---+\left(y_{n}-x_{n}\right)^{2}}=\sqrt{\sum_{i=1}^{n}\left(y_{i}-x_{i}\right)^{2}}
$$

\section{RESULTS AND ANALYSIS}

The performance comparison of proposed algorithm with other bio-inspired algorithm is illustrated in Table 2 and Table 3. Table 2 shows that the MFO algorithm always started with the highest inter-class cluster distances and Figure 5 indicate that all the algorithms performed nearly equally whenever $\mathrm{K}=4$ for all the images. Similarly the Figure 6 indicate that all the algorithms performed approximately in the same way whenever $\mathrm{K}=5$. However, the MFO algorithm gives highest fitness value and the PSO algorithm comes at the second rank. When value of cluster $K$ is $(4,5)$, MFO algorithm is better than the PSO and GA algorithm at a highest value of region based color image segmentation. Which indicates that the MFO algorithm is more effective for region based RGB color image segmentation. They show that, the proposed algorithm MFO for region based color image segmentation is the better than PSO and GA, in terms of computation times for all the images.

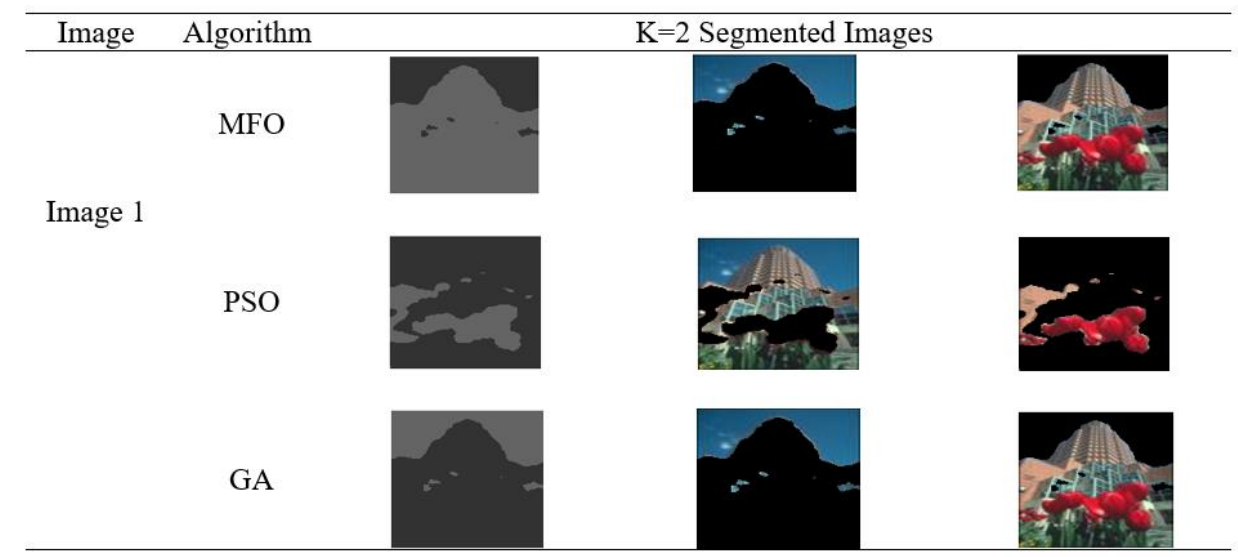

Figure 4. The region based RGB color segmented images obtained by all algorithms when the "K=2" and graph between objective function \& No. of iteration

Table 3. Computation time of all algorithms (s)

\begin{tabular}{lllll}
\hline Image & K Values & MFO & PSO & GA \\
\hline Image 1 & 5 & $13.084 \mathrm{~s}$ & $17.539 \mathrm{~s}$ & $29.460 \mathrm{~s}$ \\
& 4 & $12.857 \mathrm{~s}$ & $29.471 \mathrm{~s}$ & $25.356 \mathrm{~s}$ \\
& 3 & $10.642 \mathrm{~s}$ & $17.116 \mathrm{~s}$ & $25.760 \mathrm{~s}$ \\
& 2 & $8.777 \mathrm{~s}$ & $14.011 \mathrm{~s}$ & $21.077 \mathrm{~s}$ \\
\hline
\end{tabular}

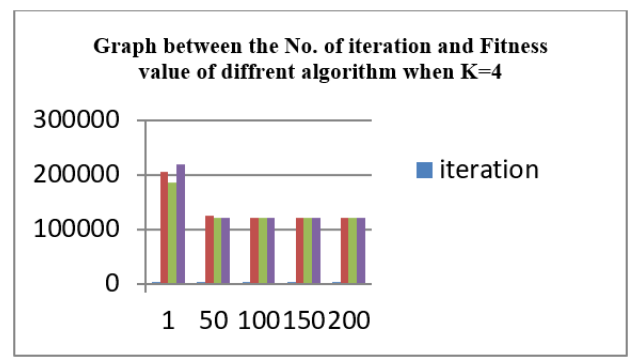

Figure 5. The graph of image 1 when $\mathrm{K}=4$ with objective value and number of iterations

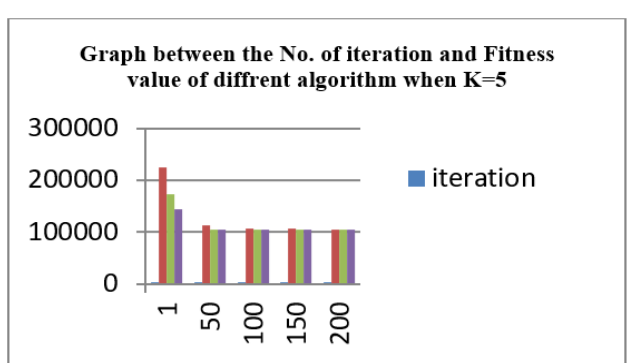

Figure 6. The graph of image 1 when $\mathrm{K}=5$ with objective value and number of iterations

\section{CONCLUSION}

The proposed method successfully developed and tested for RGB colour images datasets and obtained up to $90 \%$ cases the colour clusters. The simulation results show that the MFO algorithm is better than genetic approach, particle swarm optimization algorithms in terms of computation time and fitness value 
of segmentation of an RGB colour image. MFO concludes as an improved algorithm in terms of efficiency and speed. The algorithm tested on datasets (https://homepages.cae.wisc.edu/ece533/images) and shows their results with computation time, fitness value. Colour image segmentation using MFO algorithm can directly apply to medical image segmentation, machine-learning, content-based image retrieval system, sea object detection, and deals with the surveillance image processing which is beneficial for military purposes and several other surveillance purposes. The future work will concentrate on high-performance region based colour image segmentation by using other bio-inspired and artificial intelligence algorithms.

\section{REFERENCES}

[1] Belahbib, F., et al., "Color image segmentation by a genetic algorithm based clustering and connected component labeling," In 2012 24th International Conference on Microelectronics (ICM), pp. 1-4. IEEE, 2012.

[2] Sammouda, R., et al., "Comparison between K mean and fuzzy C-mean methods for segmentation of near infrared fluorescent image for diagnosing prostate cancer," In International Conference on Computer Vision and Image Analysis Applications, pp. 1-6, IEEE, 2015.

[3] Zhang, Y. and Donald A. A., "Prediction by partial approximate matching for lossless image compression," IEEE Transactions on Image Processing, vol. 17, no. 6, pp. 924-935, 2008.

[4] Meyer, F., "Color image segmentation," In 1992 International Conference on Image Processing and its Applications, pp. 303-306, IET, 1992.

[5] Comaniciu, D., and Peter M., "Robust analysis of feature spaces: color image segmentation," In Proceedings of IEEE computer society conference on computer vision and pattern recognition, pp. 750-755, IEEE, 1997.

[6] Velayudham A., et al., "A hybrid and novel optimization framework for denoising and classification of medical images using DTCWP and neuro-fuzzy classifiers," Journal of theoretical and applied information technology, vol. 65 , no. $1,2014$.

[7] Puranik, P., et al., "Human perception-based color image segmentation using comprehensive learning particle swarm optimization," In 2009 Second International Conference on Emerging Trends in Engineering \& Technology, pp. 630-635, IEEE, 2009.

[8] Sankari, L. "Image segmentation using glowworm swarm optimization for finding initial seed," Int. J. Sci. Res.(IJSR), vol. 3, no. 8, pp. 1611-1615, 2014.

[9] Ma, Z., and Tavares, JMRS, "A novel approach to segment skin lesions in dermoscopic images based on a deformable model," IEEE journal of biomedical and health informatics, vol. 20, no. 2, pp. 615-623, 2016.

[10] Guo, Y., et al., "An effective color image segmentation approach using neutrosophic adaptive mean shift clustering," Measurement, vol. 119, pp. 28-40, 2018.

[11] Wang, X-Y., et al., "Pixel classification based color image segmentation using quaternion exponent moments," Neural Networks, vol. 74, pp. 1-13, 2016.

[12] Cheng, S-C., "Region-growing approach to colour segmentation using 3-D clustering and relaxation labelling," IEEE Proceedings-Vision, Image and Signal Processing, vol. 150, no. 4, pp. 270-276, 2003.

[13] Scheunders, P., "A genetic c-means clustering algorithm applied to color image quantization," Pattern Recognition, vol. 30, no. 6, pp. 859-866, 1997.

[14] Li, T-HS., et al., "A fast color information setup using EP-like PSO for manipulator grasping color objects." IEEE Transactions on Industrial Informatics, vol. 10, no. 1, pp. 645-654, 2014.

[15] Belahbib, F., et al., "Color image segmentation by a genetic algorithm based clustering and connected component labeling." In 2012 24th International Conference on Microelectronics (ICM), pp. 1-4. IEEE, 2012.

[16] Rajinikanth, V., and M. S. Couceiro, "RGB histogram based color image segmentation using firefly algorithm," Procedia Computer Science, vol. 46, pp. 1449-1457, 2015.

[17] Amelio, A., and Clara P., "A genetic algorithm for color image segmentation," In European Conference on the Applications of Evolutionary Computation, Springer, Berlin, Heidelberg, pp. 314-3232013.

[18] Dong, G., and Ming X., "Color clustering and learning for image segmentation based on neural networks," IEEE Transactions on Neural Networks, vol. 16, no. 4, pp. 925-936, 2005.

[19] Neelambike S., "Comparison of K-mean and Fuzzy K-mean algorithms for color image segmentation," International Journal of Computer Science and Information Technology (IJCSIET), vol. 3, no. 3, Series 1, pp. 1-4, 2013.

[20] El Aziz, MA, Ewees AA, and Hassanien AE., "Whale optimization algorithm and moth-flame optimization for multilevel thresholding image segmentation," Expert Systems with Applications, vol. 83, pp. 242-256, 2017.

[21] Mirjalili, S., "Moth-flame optimization algorithm: A novel nature-inspired heuristic paradigm," Knowledge-Based Systems, vol. 89, pp. 228-249, 2015.

[22] Jaiswal, V., Varsha S, and Sunita V., "An implementation of novel genetic based clustering salgorithm for color image segmentation," TELKOMNIKA (Telecommunication Computing Electronics and Control), vol. 17, no. 2, pp. 1461-1467, 2019.

[23] Omran, M., Andries P.E., and Salman, A., "Particle swarm optimization method for image clustering," International Journal of Pattern Recognition and Artificial Intelligence, vol. 19, no. 03, pp. 297-321, 2015.

[24] Ganta, R.R., Zaheeruddin, S., Baddiri, N., and Rao, R.R., "Particle swarm optimization clustering based level sets for image segmentation," In 2012 Annual IEEE India Conference (INDICON), pp. 1053-1056, IEEE, 2012.

[25] Sharma, A., et al., "Recent Trends and Techniques in Image Segmentation using Particle Swarm Optimizationa Survey," International Journal of Scientific and Research Publications, vol. 5, no. 6, 2015. 